\title{
AVALIAÇÃO DOS EFEITOS AGUDOS DA PRESSÃO POSITIVA CONTINUA NAS VIAS AÉREAS EM PORTADORES DE DOENÇA PULMONAR OBSTRUTIVA CRÔNICA EXACERBADA (FASE PÓS-AGUDA)
}

\author{
Kelly de Andrade Souza Prado ${ }^{1}$, Milena de Oliveira Saltão ${ }^{1}$, Ellene Cristine Lopes ${ }^{2}$, Flavio Danilo Mungo \\ Pissulin $^{1,2}$, Claudio Spinola Najas ${ }^{1,2}$ \\ Universidade do Oeste Paulista - UNOESTE, ${ }^{1}$ Curso de Especialização em Fisioterapia Cardiorrespiratória e Hospitalar. ${ }^{2}$ Curso de \\ Fisioterapia, Presidente Prudente, SP. e-mail: pissulim@gmail.com
}

\section{RESUMO}

O presente estudo visa avaliar os efeitos agudos da pressão positiva continua nas vias aéreas (CPAP) em portadores de doença pulmonar obstrutiva crônica (DPOC) exacerbada (fase pós-aguda), assim como a influência no desfecho da internação hospitalar. Foram selecionados 30 indivíduos portadores de DPOC exacerbada, que foram orientados e submetidos ao uso da CPAP por 60 minutos, na posição semi-fowler $\left(45^{\circ}\right)$, sendo verificada a frequência respiratória (f), saturação arterial de oxigênio $\left(\mathrm{SpO}_{2}\right)$, frequência cardíaca (FC) e pressão arterial (PA) conectada a CPAP com máscara orofacial. Posteriormente, foram acompanhados para a verificação do desfecho da internação hospitalar. A média de idade dos sujeitos foi de $68,7 \pm 10,94$ anos, tendo apresentado classificação GOLD leve $10 \%$, moderado $43 \%$ e grave $47 \%$. Com relação ao desfecho da internação hospitalar foi observado que 11 sujeitos foram a óbito $(36,67 \%), 16$ indivíduos passaram por internação na UTI por um tempo médio de 4,0 $\pm 3,0$ dias. A indicação da CPAP nos portadores de DPOC exacerbada incluídos neste estudo produziu efeito agudo positivo pela melhora da $\mathrm{SpO}_{2}$. Entretanto, não houve influência no desfecho da internação hospitalar.

Palavras-chave: DPOC, exacerbada, CPAP, efeitos agudos, desfecho.

\section{EVALUATION OF ACUTE EFFECTS OF POSITIVE PRESSURE REMAINS IN THE AIRWAYS IN LUNG DISEASE CARRIERS CHRONIC OBSTRUCTIVE EXACERBATED (FASE POST - ACUTE)}

\begin{abstract}
This study aims to evaluate the acute effects of continuous positive airway pressure (CPAP) in patients with exacerbated chronic obstructive pulmonary disease (COPD; post-acute phase), as well as the influence on the outcome of hospitalization. A total of 30 patients with exacerbated COPD were selected, oriented and submitted to the use of CPAP for 60 minutes in the semi-fowler position $\left(45^{\circ}\right)$, have been checked the respiratory rate (f), arterial oxygen saturation $\left(\mathrm{SpO}_{2}\right)$, heart rate $(\mathrm{HR})$ and arterial pressure (AP) connected to CPAP with orofacial mask. Later, they were accompanied for checking the outcome of hospitalization. The patients mean age was $68.7 \pm 10.94$ years and showed the following classifications: GOLD $10 \%$, moderate $43 \%$ and serious $47 \%$. Regarding the outcome of hospitalization, it has been observed that 11 individuals died (36.67\%), 16 were admitted to the ICU for an average of $4.0 \pm 3.0$ days. The indication of CPAP in patients with exacerbated COPD, included in this study, produced positive acute effect by the improvement of $\mathrm{SpO}_{2}$. However, there was no influence in the outcome of hospitalization.
\end{abstract}

Keywords: COPD, exacerbated, CPAP, acute effects, outcome.

\section{INTRODUÇÃO}

A doença pulmonar obstrutiva crônica (DPOC) é considerada como uma síndrome por obstrução crônica ao fluxo aéreo decorrente a uma bronquite crônica ou enfisema ${ }^{1}$.

Estima-se que no Brasil, entre 3 e 7 milhões de brasileiros tenham DPOC. Segundo dados do DATASUS (Departamento de
Informática do Sistema Único de Saúde), no ano de 2010 a DPOC gerou no Sistema Nacional de Saúde Pública, 141.994 hospitalizações que levaram a 778.428 dias de internação. O custo total dessas internações foi de $\mathrm{R} \$ \mathbf{9 2 . 4 3 4 . 4 1 5 , 5 1}$ e houve 7.937 mortes diretamente relacionadas com esta patologia. A prevalência de DPOC no Brasil segundo a Sociedade Brasileira de 
Pneumologia e Tisiologia (SBPT) é de 15,8\% em adultos acima de 40 anos $^{2}$.

A bronquite crônica provoca modificações dos brônquios, onde possuem definições bem características como presença de tosse e produção de expectoração por menos de três meses em dois anos consecutivos, e a destruição do parênquima pulmonar causará a enfisema, tendo como consequência uma diminuição da sua elasticidade e surgindo os sintomas de dispneia aos esforços ${ }^{3}$.

A ventilação não invasiva (VNI) consiste na aplicação de uma pressão positiva, por meio de máscaras nasais ou faciais, fazendo uma melhora na troca gasosa por recrutamento de alvéolos hipoventilados e diminuindo o trabalho dos músculos respiratórios. O uso da VNI pode manter as barreiras de defesa natural, o que evita complicações e reduz o período de internação hospitalar e da Unidade de terapia intensiva (UTI), além de possibilitar redução no uso de sedação e menor prevalência de entubação orotraqueal com as suas complicações ${ }^{4}$.

Uma das modalidades de VNI é a pressão positiva continua nas vias aéreas (CPAP), a qual consiste na manutenção de uma pressão positiva durante todo o ciclo respiratório ${ }^{5}$.

Baseia-se no princípio que envolve a diminuição do recrutamento de músculos inspiratórios, menor trabalho elástico, prevenção do colabamento dinâmico das vias aéreas e menor trabalho resistivo ${ }^{6}$. Já foi observado diminuição da mortalidade e custos de tratamentos, com o uso da modalidade BIPAP (Pressão positiva nas vias aéreas por dois níveis pressóricos) em insuficiência respiratória aguda, motivo pelo qual o seu uso vem se tornando cada vez mais frequente ${ }^{7}$.

Há estudos que demostraram que o uso da CPAP em pacientes com DPOC exacerbada com a pressão positiva ao final da expiração (PEEP) de 10-12 $\mathrm{cmH}_{2} \mathrm{O}$, diminui o trabalho respiratório e a dispnéia ${ }^{7,8}$.

Um estudo mostrou que com a utilização da VNI com pressão positiva continua nas vias aéreas (CPAP) em portadores de DPOC exacerbada promoveu menor incidência de pneumonia nosocomial de $17 \%$ para $5 \%$, comparado ao tratamento convencional, também redução da necessidade de entubação de $74 \%$ para $26 \%$ e redução da mortalidade de $29 \%$ para $9 \%{ }^{10}$.

Outros estudos têm demostrado que a indicação da CPAP reduz o tempo de permanência na unidade de terapia intensiva (UTI), o tempo de internação hospitalar, a necessidade de intubação traqueal, a ocorrência de pneumonia associada à ventilação mecânica, reduz o número de complicações, alivia sintomas como dispnéia ocasionada pela fadiga da musculatura respiratória, diminui a $\mathrm{PaCO}_{2}$ (pressão arterial de gás carbônico) e reduz a mortalidade de pacientes com insuficiência respiratória por exacerbação da DPOC ${ }^{11,12}$.

Assim a ventilação não invasiva (VNI) é uma alternativa terapêutica para pacientes com agudização da DPOC ${ }^{13}$.

Está bem estabelecida a relevância do uso da pressão positiva bifásica nas vias aéreas (BIPAP) nos portadores de DPOC exacerbada. Entretanto, na fase pós-aguda ainda durante a internação hospitalar, estes pacientes podem desenvolver um quadro de insuficiência respiratória aguda. Por isso, o uso da VNI com a modalidade CPAP necessita ser investigado.

$O$ objetivo do presente estudo foi avaliar os efeitos agudos da CPAP em portadores de DPOC exacerbada (fase pós-aguda), assim como a influência da técnica no desfecho da internação hospitalar.

\section{MÉTODOS}

O estudo foi realizado num hospital público de Presidente Prudente - São Paulo. Foi avaliado e aprovado pelo Comitê de Ética em Pesquisa da Unoeste (CAAE 24691913.7.0000.5515). Após esclarecimento, os voluntários assinaram um termo de consentimento livre.

Foram selecionados 30 voluntários portadores de DPOC em fase pós-aguda de exacerbação. A ausência de dados epidemiológicos sobre $\mathrm{o}$ atendimento desta população na referida unidade hospitalar dificultou o cálculo amostral, apesar da prevalência da DPOC em geral (estável e exacerbada) já ter sido descrita. Por isso, foi levado em consideração o número de leitos disponíveis e o tempo de coleta de dados. O CPAP foi aplicado por $60 \mathrm{~min}$, verificando os parâmetros ventilatórios e hemodinâmicos durante este período, além da observação dos mesmos antes e após a aplicação da técnica. Com isso, foi possível verificar os efeitos agudos da técnica.

Os portadores de DPOC que foram incluídos no Grupo de Estudo (GE) eram 
conscientes, orientados, com frequência respiratória (f) $<28 \mathrm{rpm}$, saturação arterial de oxigênio $\left(\mathrm{SpO}_{2}\right)>80 \%$ por oximetria de pulso, frequência cardíaca $(\mathrm{FC})$ e pressão arterial (PA) que demonstram estabilidade hemodinâmica, em fase pós-aguda de exacerbação. Como critério de exclusão, foram considerados rebaixamento do nível de consciência e/ou desorientação, $\mathrm{SpO}_{2}<$ $80 \%$ e instabilidade hemodinâmica observada pela FC e PA ${ }^{22}$. Com estes critérios de inclusão e exclusão houve homogeneização do grupo estudado.

A modalidade de VNI usada neste estudo foi o CPAP, utilizando o reanimador de Muller (Marca Engesp), que possui uma válvula reguladora de pressão, um circuito com gatilho que isolamos, venturi, copo de nebulização, e máscara facial (Marca Image 3), fixando-a com uma presilha cefálica. A pressão utilizada foi padronizada de acordo com o conforto respiratório relatado pelo paciente, variando entre 5 e $12 \mathrm{cmH}_{2} \mathrm{O}$.

Avaliamos a severidade do grau de obstrução com o a classificação GOLD. Segundo GOLD a DPOC é classificada em 4 estágios de severidade na dependência do grau de obstrução das vias aéreas medido pelo volume expiratório forçado: I- Leve, II- Moderado e III- Grave ${ }^{14}$.

Os pacientes foram submetidos aos seguintes procedimentos: (1) posicionamento em semi-fowler $\left(45^{\circ}\right)$, (2) verificação da $f \mathrm{com}$ observação da oscilação da caixa torácica, da $\mathrm{SpO}_{2}$ e da $\mathrm{FC}$ com oximetro de pulso (marca Dixtal), e da PA com esfigmomanômetro (marca Bic), (3) conexão da CPAP com máscara orofacial, (4) verificação dos dados do item 2 antes $(10 \mathrm{~min}$, M1), durante (10 min de aplicação, M2; 50 min de aplicação, M3 com total de $60 \mathrm{~min}$ ) e depois da aplicação da VNI (30 min, M4).

Para avaliar o desfecho da internação hospitalar, os pacientes foram acompanhados para a verificação dos dias de internação hospitalar, retorno pra UTI, alta hospitalar ou óbito. Após nosso atendimento estes pacientes continuaram a ser atendidos pelo serviço de fisioterapia do hospital, dando continuidade ao uso da CPAP.

Todos os parâmetros mensurados para verificar os efeitos agudos da CPAP e aqueles empregados para verificar o desfecho da internação hospitalar, foram devidamente registrados em uma ficha de avaliação.

Para análise dos dados, comparação entre os momentos foi utilizada ANOVA, para modelos de medidas repetidas, sendo complementada com os testes de Tukey, considerando o nível de 5\% de significância.

\section{RESULTADOS}

Foram incluídos 30 indivíduos (19 homens e 11 mulheres) portadores de DPOC, cuja média de idade e classificação GOLD podem ser observadas na Tabela 1.

Tabela 1. Média e desvio padrão das características gerais dos pacientes incluídos no estudo.

\begin{tabular}{lc}
\hline Características & Média \\
\hline Idade (anos) & $68,07 \pm 10,94$ \\
\hline Tempo tabagismo (anos) & $50,14 \pm 13,69$ \\
\hline $\begin{array}{l}\text { Estimativa do número de maços } \\
\text { consumidos/dia }\end{array}$ & $1,77 \pm 0,95$ \\
\hline $\begin{array}{l}\text { Estimativa do número de cigarros } \\
\text { consumidos/dia }\end{array}$ & $34,6 \pm 20,14$ \\
\hline GOLD leve (\%) & 10 \\
\hline GOLD moderado (\%) & 43 \\
\hline GOLD grave (\%) & 47 \\
\hline GOLD: Global Initiative for Chronic obstructive Lung Disease.
\end{tabular}

Não foram verificadas diferenças estatísticas entre os momentos para as médias das variáveis FC e PAD. A média da $f$ aferida em M1 foi estatisticamente superior a verificada em $\mathrm{M} 3$ e M4. A média da $\mathrm{SpO}_{2}$ aferida em $\mathrm{M} 1$ foi estatisticamente inferior aos registros em M1, M2 e M3. Este mesmo parâmetro foi estatisticamente superior nos momentos $\mathrm{M} 2$ e M3 em relação ao observado em M4. A PAS média verificada em M2 foi estatisticamente superior à observada em M3 (Tabela 2).

Com relação ao desfecho da internação hospitalar os resultados podem ser observados na Tabela 3. 
Tabela 2. Médias e desvio padrões dos parâmetros frequência cardíaca, frequência respiratória, saturação, pressão arterial sistólica e pressão arterial diastólica.

\begin{tabular}{lccccc}
\hline Parâmetro & M1 & M2 & M3 & M4 & P \\
\hline $\mathrm{FC}$ & $83,9 \pm 17,8 \mathrm{a}$ & $81,4 \pm 16,3 \mathrm{a}$ & $84,7 \pm 18,9 \mathrm{a}$ & $84,0 \pm 18,5 \mathrm{a}$ & 0,3650 \\
\hline $\mathrm{FR}$ & $21,9 \pm 3,9 \mathrm{a}$ & $21,1 \pm 3,3 \mathrm{ab}$ & $20,1 \pm 3,0 \mathrm{~b}$ & $20,0 \pm 2,7 \mathrm{~b}$ & 0,0005 \\
\hline $\mathrm{SpO} \mathrm{H}_{2}$ & $91,5 \pm 3,7 \mathrm{a}$ & $96,3 \pm 2,6 \mathrm{~b}$ & $96,6 \pm 2,6 \mathrm{~b}$ & $94,0 \pm 3,0 \mathrm{c}$ & 0,0001 \\
\hline $\mathrm{PAS}$ & $132,3 \pm 28,7 \mathrm{ab}$ & $137,2 \pm 24,4 \mathrm{a}$ & $130,2 \pm 25,2 \mathrm{~b}$ & $131,6 \pm 27,5 \mathrm{ab}$ & 0,0235 \\
\hline $\mathrm{PAD}$ & $82,3 \pm 14,8 \mathrm{a}$ & $82,3 \pm 13,4 \mathrm{a}$ & $77,0 \pm 12,2 \mathrm{a}$ & $77,3 \pm 13,0 \mathrm{a}$ & 0,1369 \\
\hline
\end{tabular}

* Letras diferentes na mesma linha representam diferenças estatisticamente significativas nos diferentes momentos: M1 - antes de 10 min da aplicação da VNI, M2 - após 10 min da aplicação da VNI, M3 - após 50 min da aplicação da VNI e M4 - após 30 min do termino da aplicação da VNI.

Tabela 3. Variáveis do desfecho da internação hospitalar.

\begin{tabular}{lc}
\hline Parâmetro & Total \\
\hline Óbito & $11 / 30(36,67 \%)$ \\
\hline Internação em UTI & $16 / 30(53,33 \%)$ \\
\hline Tempo médio de uso da UTI (dias) & $4,0 \pm 3,0$ \\
\hline Tempo médio de internação & $24,9 \pm 25,2$ \\
\hline
\end{tabular}

\section{DISCUSSÃO}

A DPOC vem trazendo grande interesse e preocupação da comunidade cientifica por seu comportamento epidemiológico de crescente prevalência e mortalidade. Sendo assim, tornamse cada vez mais necessários o diagnóstico precoce e o gerenciamento adequado da doença para reduzir os impactos econômico e social causados por ela. Mesmo sabendo do crescente impacto, ela continua a ser sub-reconhecida e/ou subdiagnosticada, levando ao início tardio do tratamento e, com isto, à perda de oportunidades para evitar a piora da doença ${ }^{15,16}$.

Os pacientes portadores de DPOC desenvolvem, frequentemente, exacerbações que provocam grande impacto clínico e à utilização de serviços de saúde. No ano de 2011 a DPOC já foi constituída a quarta causa mais frequente de mortalidade e âmbito mundial ${ }^{17,18}$. Os principais fatores de custo na DPOC são exacerbações graves que necessitam de internação hospitalar e, constantemente, estão associados ao aumento da mortalidade e à redução da qualidade de vida ${ }^{19}$.

O presente estudo verificou que 19 pacientes $(63,33 \%)$, que usaram a VNI com CPAP, sobreviveram ao período de exacerbação. Destes, 14 pacientes $(46,67 \%)$ estavam internados em enfermarias e 16 pacientes $(53,33 \%)$ passaram por internação na UTI. Foram a óbito 11 pacientes (36,67\%). Alguns estudos demonstraram que a incidência de óbito foi de
$40 \%$, nos pacientes que foram ventilados mecanicamente ${ }^{19,20}$, parecido com o que foi observado no presente estudo.

No Brasil ocorreu um aumento do número de óbitos por DPOC nos últimos 20 anos, tanto no sexo masculino quanto feminino, tendo a taxa de mortalidade de 7,88 em cada 100.000 habitantes na década de 1980, para cada 19,04 em cada 100.000 habitantes na década de 1990 mostrando um aumento significativo de óbitos por DPOC de 1980 até 2001, chegando um percentual de $340 \%{ }^{21}$.

Aproximadamente $1 / 3$ dos pacientes com exacerbação aguda da DPOC morreram durante a internação na UTI e a mortalidade ainda se mantem alta no primeiro ano após a alta hospitalar. Neste estudo observaram que a mortalidade foi de $27,3 \%$ durante a internação na UTI, com incidência de óbito menor que a observada em nosso estudo ${ }^{17-19}$. Como, no presente estudo, não foi observado o seguimento anterior a internação dos portadores de DPOC exacerbado selecionados, é possível que alguns destes indivíduos já apresentavam admissões hospitalares anteriores, tinham acidose respiratória ou necessitaram de suporte ventilatório invasivo, o que influencia diretamente no resultado.

A média de idade dos pacientes incluídos em nosso estudo foi de $68,07 \pm 10,94$ anos, o que representa um fator de risco para a má evolução da agudização ${ }^{22}$. Este fato também pode explicar a prevalência de óbito encontrada.

Também em nosso estudo foi avaliado a classificação GOLD (The Global Iniciative for Chronic Lung Disease), demonstrando que 10\% apresentaram DPOC leve, $43 \%$ moderado e $47 \%$ grave. De acordo com a classificação GOLD a exacerbação da DPOC é definida como um evento agudo no curso natural da doença, classificando leve, moderado e grave, pacientes com VEF1 < 
$50 \%$ do previsto tem seis vezes mais chances de sofrer uma exacerbação, comparando aos pacientes que apresentam uma VEF1 > que $50 \%{ }^{14}$.

Estudos demonstraram que a indicação da CPAP é benéfica na redução do tempo de permanência na unidade de terapia intensiva (UTI), do tempo de internação hospitalar, na necessidade de intubação traqueal, na ocorrência de pneumonia associada à ventilação mecânica, na redução do número de complicações, no alívio de sintomas como dispnéia ocasionada pela fadiga da musculatura respiratória, na diminuição da $\mathrm{PaCO}_{2}$ (pressão arterial de gás carbônico) e na redução na mortalidade de pacientes com insuficiência respiratória por exacerbação da DPOC $^{11,12}$.

Não foi possível, neste estudo, estabelecer que houve redução dos dias de internação hospitalar ou da incidência de óbito com o uso da CPAP. Entretanto, a elevação significativa da $\mathrm{SpO}_{2}$, mesmo após a remoção da CPAP, demonstrou que há efeito agudo positivo, o que pode ter reduzido a sensação de dispneia dos pacientes estudados.

\section{CONCLUSÃO}

Conclui-se que a indicação da CPAP nos portadores de DPOC exacerbado incluídos neste estudo, produziu efeito agudo positivo pela melhora da $\mathrm{SpO}_{2}$. Entretanto, não houve influência no desfecho da internação hospitalar.

\section{CONFLITO DE INTERESSE}

Os autores declaram não haver qualquer potencial de conflito de interesse que possa interferir na imparcialidade deste trabalho científico.

\section{REFERÊNCIAS}

1. Buist AS. Gold (Global Initiative for Chronic Obstructive Lung Disease). Global Strategy for the Diagnosis, Management, and Prevention of Chronic Obstructive Pulmonary Disease 2006;01-88. Disponível em: http://www.goldcopd.com

2. Jardim JR, Oliveira JA, Nascimento O. Consenso Brasileiro sobre DPOC. Revisão de alguns aspectos de epidemiologia e tratamento da doença estável. Brasil: Sociedade Brasileira de Pneumologia e Tisiologia; 2006.
3. Consenso Brasileiro sobre Doença Pulmonar Obstrutiva Crônica II - DPOC. J Bras Pneumol. 2004;30(Supl 5):S1-42.

4. Conti G, Costa R, Craba A, Festa V, Catarci, S. Noninvasive ventilation in COPD patients. Minerva Anestesiol. 2004;70:145-50.

5. Denehy L, Berney S. The use of positive pressure devices by phisiotheapists. Eur Respi J. 2001;17:821-9. DOI:

http://dx.doi.org/10.1183/09031936.01.17408210

6. HOO GWS. Nonpharmacologic adjunts to traininig during pulmonary rehabilitation: The role of supplemental oxygen and noninvasive ventilation. J Rehab Res Developm. 2003;40(5): 81-98.

7. Schettino GP, Reis MA, Galas F, Park M, Franca SA, Okamoto VN, et al. Ventilação mecânica não-invasiva com pressão positiva. Rev Bras Ter Intens. 2007;19(2):245-57.

DOI:

http://dx.doi.org/10.1590/\$0103-

507X2007000200019

8. Goldberg P, Reissmann H, Maltais F, et al. Effi cacy of noninvasive CPAP in COPD with acute respiratory failure. Eur Respir J. 1995;8:1894-1900. DOI: http://dx.doi.org/10.1183/09031936.95.08111894

9. O'Donoghue FJ, Catcheside PG, Jordan AS, et al. Effect of CPAP on intrinsic PEEP, inspiratory effort, and lung volume in severe stable COPD. Thorax. 2002;57:533-9.

DOI:

http://dx.doi.org/10.1136/thorax.57.6.533

10. Muhoz AM, Aldrighi CM, Buschpiegel C, Ono C, Montag E, Fells $\mathrm{K}$ et al. The feasibility of sentinela lymph node detection patients with previous transaxillary implant breast augmentation: preliminary results. Aesth Plast Surg. 2005;29(3):163-8. DOI: http://dx.doi.org/10.1007/s00266-004-0103-8

11. Celikel T, Sungur M, Ceyhan B, et al. Comparison of noninvasive positive pressure ventilation with standard medical therapy in hypercapnic acute respiratory failure. Chest. 1998;114:1636-42. DOI: http://dx.doi.org/10.1378/chest.114.6.1636

12. Neme JY, Gutierrez AM, Santos MC, et al. Physiologic effects of noninvasive ventilation in patients with chronic obstructive pulmonary disease. Arch Bronconeumol. 2007;43:150-5. DOI: http://dx.doi.org/10.1157/13099531

13. Campos LEM. Doença pulmonar obstrutiva crônica (DPOC). In: Petroianu A. Urgências clínicas e cirúrgicas. Rio de Janeiro: Guanabara-Koogan; 2002.

14. Gold. Global Strategy for The Diagnosis, Management, And Preventio of Chronic obstructive pulmonary disease (Revised 2011). Washington: Global Initiative for Chronic obstructive Lung Disease; 2011.

15. Mannino DM, Braman S. The epidemiology and economics of chronic obstructive pulmonary disease. Proc Am Thorac Soc. 2007;4(7):502-6. DOI: http://dx.doi.org/10.1513/pats.200701-001FM

16.Takahashi T, Ichinose M, Inoue H, Shirato K, Hattori $\mathrm{T}$, Takishima T. Underdiagnosis and undertreatment of 
COPD in primary care settings. Respirology. 2003;8(4):504-8.

DOI:

http://dx.doi.org/10.1046/i.1440-1843.2003.00501.x

17. Ai- Ping C, Lee KH, Lim TK. In-hospital and 5-year mortality of patients treated in the ICU for acute exacerbation of COPD: a retrospective study. Chest. 2005,128(2):518-24.

DOI:

http://dx.doi.org/10.1378/chest.128.2.518

18. Quinnell TG, Pilsworth S, Shneerson JM, Smithe IE. Prolonged invasive ventilation following acute ventilator failure in COPD: weaning results, survival, and the role of noninvasive ventilation. Chest. 2006;129(1):133-9.

DOI:

http://dx.doi.org/10.1378/chest.129.1.133

19. Connors AF Jr, Dawson NV, Thomas C, Harrell FE Jr, Desbiens N, Fulkerson J et al. Outcomes following acute exacerbation of severe chronic obstructive lung disease. The SUPPORT investigators (Study to Understand Prognoses and Preferences for Outcomes and Risks of Treatments). Am J Respir Crit Care Med. 1996;154(4 Pt 1):959-67. Erratum in: Am J Respir Crit Care Med. 1997;155(1):386. DOI: http://dx.doi.org/10.1164/ajrccm.154.4.8887592

20. Johannessen A, Nilsen R. M, Storebo M, Gulsvik A, Eagan T, et al. Comparison of 2011 and 2007 Global Initiative for Chronic Obstructive Lung Disease Guidelines for Predicting Mortality and Hospitalalization. 2007. Disponível em: http://www.pneumoatual.com.br

21. Jardim J, Oliveira J, Nascimento O. II Consenso de DPOC da Sociedade Brasileira e Pneumologia e Tisiologia. J Bras Pneumol. 2004;30(supl 5)S1-S41.

22. Ram FS, Picot J, Lightowler J, Wedzicha JA. Noninvasive positive-pressure ventilation for treatment of respiratory failure due to exacerbations of chronic obstructive pulmonary disease. Cochrane Database Syst Rev. 2004;(3):CD004104.

Recebido para publicação em 20/07/2015

Revisado em 24/07/2015

Aceito em 27/07/2015 